\title{
Syntheses of Optically Active 2,3,6-Tri- $O$-benzyl-D-myo-inositol, Laminitol, and Mytilitol from D-Glucose
}

\author{
Ken-ichi Sato, ${ }^{*}$ Masayuki BoKura, and Makoto Taniguchi \\ Laboratory of Organic Chemistry, Faculty of Engineering, Kanagawa University, \\ Rokkakubashi, Kanagawa-ku, Yokohama 221 \\ (Received September 24, 1993)
}

\begin{abstract}
2,3,6-Tri-O-benzyl-D-myo-inositol, which is a key intermediate of D-inositol 1,4,5-triphosphate, was synthesized from $\mathrm{D}$-glucose without performing any optical resolution by utilizing $C_{2}$ symmetry. Laminitol and mytilitol were also synthesized from $\mathrm{D}$-glucose via the same key intermediate, $1 \mathrm{D}-(1,3 / 2,4)$-tetra- $O$-benzyl-2- $C$ methyl-5-cyclohexene-1,2,3,4-tetrol.
\end{abstract}

Since the discovery of the role of D-myo-inositol 1,4 , 5 -triphosphate $\left(\mathrm{IP}_{3}, \mathbf{1}\right)$ as an intracellular second messenger for calcium mobilization, ${ }^{1)}$ the biological interest in $\mathrm{IP}_{3}$ has greatly increased. In order to explore the biochemical process, a simple, general, and efficient methodology for the chemical syntheses of 1 and its derivatives is required that will act as agonists in the phosphoinositide system. ${ }^{2)}$ The biological importance of other stereoisomers of inositol is less well established. ${ }^{3)}$ However, there are two known naturally-occurring $C$ methyl inositols, both isolated from algae: (-)-laminitol (3) and mytilitol (4) (Chart 1). Compound $\mathbf{3}$ is especially interesting in that it has the myo-inositoltype configuration with a methyl group at $C-4$, and that it inhibits the growth of Neurospora crassa. ${ }^{4)}$ Syntheses of racemic laminitol from myo-inositol ${ }^{5)}$ and (-)laminitol from toluene ${ }^{6)}$ have been reported. To date, however, for the syntheses of $\mathbf{1}$ and its derivatives, naturally occurring cyclitol derivatives, myo-inositol, ${ }^{7}$ ) quebrachitol, ${ }^{8)}$ and quinic acid ${ }^{9)}$ have been mainly used as the starting materials. In general, optical resolution has been required in the case of using myo-inositol, which has a plane of symmetry. We now report on novel synthetic methods of optically active laminitol and mytilitol, as well as details concerning a communication $^{10)}$ about 2,3,6-tri- $O$-benzyl-D-myo-inositol (2) from D-glucose, respectively.

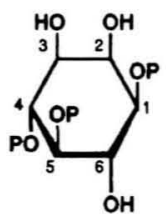

D-myo-inositol 1,4,5-triphosphate, 1

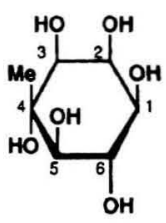

Laminitol, 3

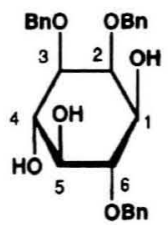

2

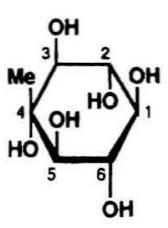

Mytilitol, 4
Chart 1 .

\section{Results and Discussion}

Methyl 3,4-bis( $O$-methoxymethyl)-2,6-bis[ $O$ - $(p$-toly]sulfonyl)]- $\alpha$-D-glucopyranoside (5) was prepared from D-glucose in $56 \%$ yield (3 steps) (Scheme 1). 6-Deoxyhex-5-enopyranoside derivative (6) was synthesized by the treatment of $\mathbf{5}$ with sodium iodide, tetrabutylammonium iodide, 1,8-diazabicyclo[5.4.0]undec-7-ene (DBU), and molecular sieves $4 \mathrm{~A}$ in dimethyl sulfoxide (DMSO) at $90{ }^{\circ} \mathrm{C}$ (one-pot reaction, $63 \%$ yield). Detosylation of $\mathbf{6}$, followed by protection with a benzyl group, gave methyl 2- $O$-benzyl-6-deoxy-3,4-bis $(O$ methoxymethyl)- $\alpha$-D-xylo-hex-5-enopyranoside (8) in $87 \%$ yield (2 steps). A Ferrier reaction of 8 gave a partially protected 2,3,4,5-tetrahydroxycyclohexanone derivative, which was treated with acetic anhydride in pyridine to give the corresponding enone derivative (9) in $77 \%$ yield ( 2 steps). The reduction of 9 with sodium tetrahydroborate-cerium (III) chloride in dichloromethane-ethanol $(1: 2)$, followed by benzylation of the hydroxyl group gave the protected cyclohexenol derivative (10) in $89 \%$ yield (2 steps). Oxidation of $\mathbf{1 0}$, which has a $C_{2}$ symmetry axis, ${ }^{11)}$ with osmium tetraoxide gave a partially protected myo-inositol derivative (11) in $83 \%$ yield. The regioselective protection of the vicinal hydroxyl groups of $\mathbf{1 1}$ by using dibutyltin oxide and chloromethyl methyl ether, followed by benzylation of the remaining hydroxyl group, gave the fully protected myo-inositol derivative (13) in $79 \%$ yield (2 steps). Hydrolytic removals of the methoxymethyl groups of $\mathbf{1 3}$ gave 2,3,6-tri- $O$-benzyl-D-myo-inositol 2 in $90 \%$ yield.

In a similar manner as mentioned above, $C$-methyl inositols, (-)-laminitol and mytilitol, were synthesized via $4-C$-methyl-branched 6 -deoxyhex-5-enopyranoside. ${ }^{12)}$ First, methyl 2,3-di- $O$-benzyl-6- $O$-triphenylmethyl- $\alpha$-D-glucopyranoside was prepared from D-glucose in $32 \%$ yield ( 5 steps). The above-mentioned compound was oxidized ${ }^{13)}$ to give the corresponding 4 - ulose $^{14)}$ in $86 \%$ yield (Scheme 2). Compound (15) was prepared via 14 in $56 \%$ yield (2 steps) by treating the above-mentioned 4-ulose with methyllithium at $-78{ }^{\circ} \mathrm{C}$, followed by benzylation. Selective hydrolysis, followed by tosylation of $\mathbf{1 5}$, gave the $6-O-(p$-tolylsulfonyl) derivative (17) in quantitative yield (2 steps). 

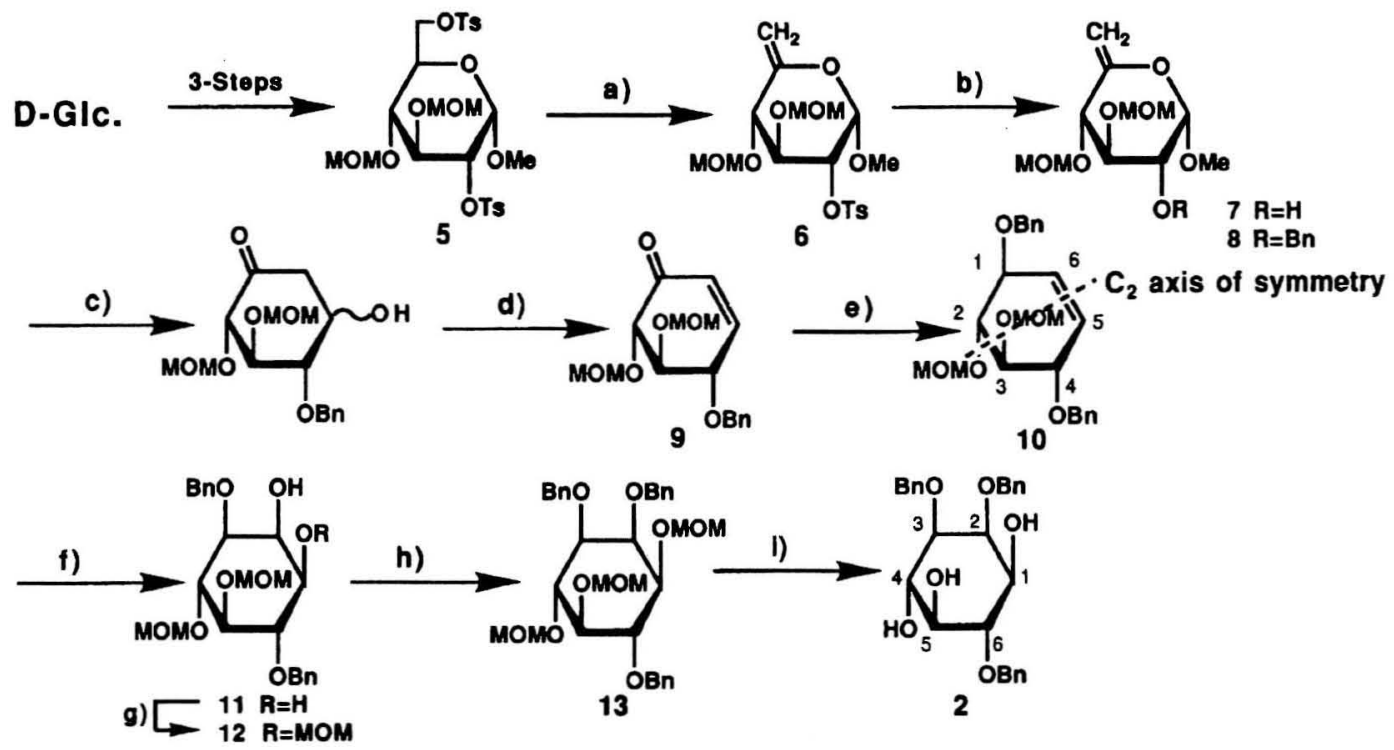

a) $\mathrm{NaI}, \mathrm{Bu}_{4} \mathrm{NI}$, then $\mathrm{DBU} / \mathrm{DMSO}, 90{ }^{\circ} \mathrm{C}, 63 \%$. b) $\mathrm{NaOMe} / \mathrm{MeOH}$, reflux, then $\mathrm{NaH}, \mathrm{BnBr} / \mathrm{DMF}, 0{ }^{\circ} \mathrm{C}, 87 \%$. c) $\mathrm{Hg}(\mathrm{OAc})_{2}$ /acetone- $\mathrm{H}_{2} \mathrm{O}, 1 \% \mathrm{AcOH}$, reflux, $77 \%$. d) $\mathrm{Ac} 2 \mathrm{O} /$ pyridine, r.t., quant. e) $\mathrm{NaBH}_{4}, \mathrm{CeCl}_{3} \cdot 7 \mathrm{H}_{2} \mathrm{O} / \mathrm{CH}_{2} \mathrm{Cl} 2-\mathrm{EtOH}$ $(1: 2),-78{ }^{\circ} \mathrm{C}$, then $\mathrm{NaH}, \mathrm{BnBr} / \mathrm{DMF}, 0{ }^{\circ} \mathrm{C}, 89 \%$. f) $\mathrm{OsO}_{4}, 4$-methylmorpholine $\mathrm{N}$-oxide/acetone- $\mathrm{H}_{2} \mathrm{O}$ (4:1), r.t., $83 \%$. g) $\mathrm{Bu}_{2} \mathrm{SnO} / \mathrm{C}_{6} \mathrm{H}_{6}$, reflux, then $\mathrm{MOMCl}, \mathrm{Et}_{3} \mathrm{~N} / \mathrm{C}_{6} \mathrm{H}_{6}$, r.t., $82 \%$. h) $\mathrm{NaH}, \mathrm{BnBr} / \mathrm{DMF}, 0{ }^{\circ} \mathrm{C}, 96 \%$. i) $0.1 \mathrm{M} \mathrm{HCl}-\mathrm{MeOH}, 63$ ${ }^{\circ} \mathrm{C}, 90 \%$

Scheme 1.
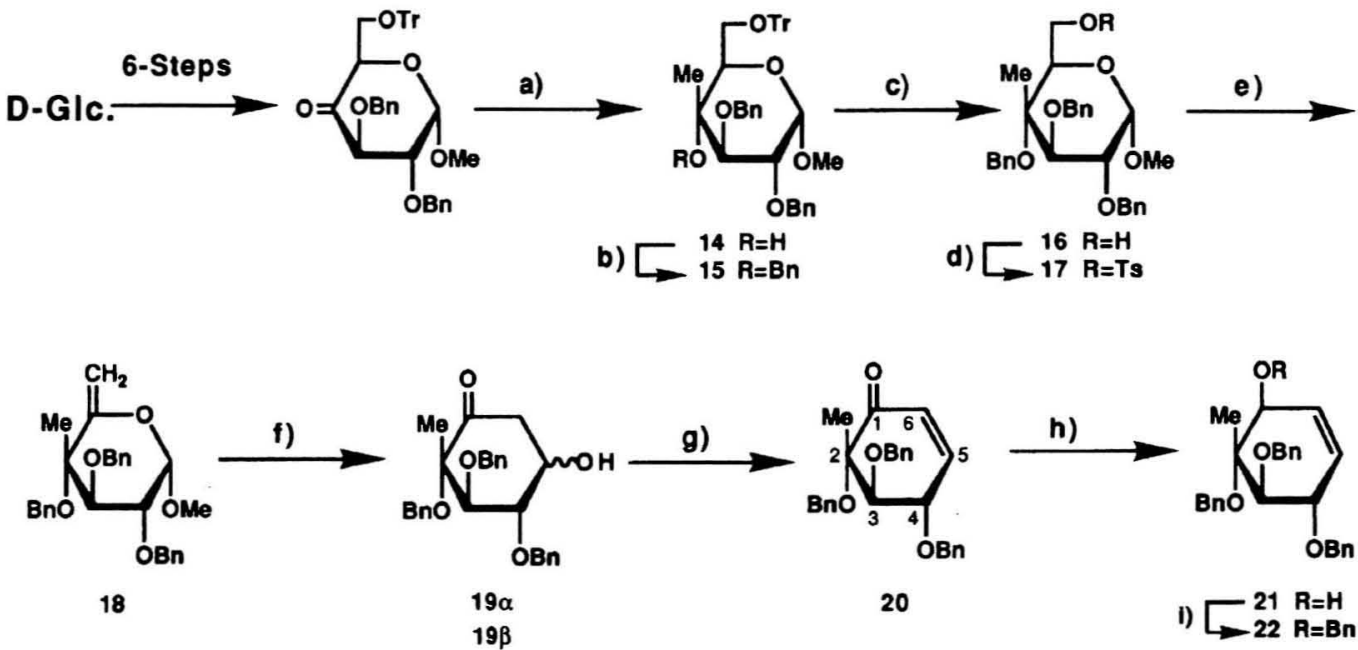

a) $\mathrm{MeLi} / \mathrm{Et}_{2} \mathrm{O},-78{ }^{\circ} \mathrm{C}, 72 \%$. b) $\mathrm{NaH}, \mathrm{BnBr} / \mathrm{DMF}, 0{ }^{\circ} \mathrm{C}, 78 \%$. c) $70 \% \mathrm{AcOH}, 70{ }^{\circ} \mathrm{C}$, quant. d) TsCl/Py., r.t., $99 \%$. e) $\mathrm{NaI}, \mathrm{Bu}_{4} \mathrm{NI}$, then $\mathrm{DBU} / \mathrm{DMSO}, 120{ }^{\circ} \mathrm{C}, 61 \%$. f) $\mathrm{HgCl}_{2} / \mathrm{H}_{2} \mathrm{O}$-Acetone (2:5), reflux, $72 \%$. g) $\mathrm{Ac} 2 \mathrm{O} / \mathrm{Py}$., r.t., $94 \%$. h) $\mathrm{NaBH}_{4}, \mathrm{CeCl}_{3} 7 \mathrm{H}_{2} \mathrm{O} / \mathrm{CH}_{2} \mathrm{Cl}_{2}-\mathrm{EtOH}(1: 2),-78^{\circ} \mathrm{C}, 91 \%$. i) $\mathrm{NaH}, \mathrm{BnBr} / \mathrm{DMF}, 0{ }^{\circ} \mathrm{C}$, quant.

Scheme 2.

Compound $\mathbf{1 7}$ was transformed into the corresponding 5 -enopyranoside (18) in $61 \%$ yield by the use of $\mathrm{NaI}, \mathrm{Bu}_{4} \mathrm{NI}, \mathrm{DBU}$, and MS $4 \mathrm{~A}$ in DMSO (one-pot reaction). ${ }^{14)}$ The $C$-methyl-branched 2,3,4,5-tetrahydroxycyclohexanone derivative $(\mathbf{1 9})^{12)}$ was prepared from $\mathbf{1 8}$ by using Ferrier's method. ${ }^{15)}$ Then, 19 was treated with acetic anhydride in pyridine to give $\mathbf{2 0}$ in $68 \%$ yield ( 2 steps). A selective reduction of the carbonyl group of $\mathbf{2 0}$ and benzylation of a newly introduced hydroxyl group gave the corresponding cyclohexene derivative $(22)$ in $91 \%$ yield ( 2 steps). The configuration at the hydroxyl group of $\mathbf{2 1}$ was presumed by considering the stereoselectivity in the reduction of $\mathbf{9}$, and was further proved to derive to the known compound 3 as follows. The oxidation of $\mathbf{2 2}$ with osmium tetraoxide gave a mixture of compounds 23 and 24 in a ratio of $3: 5$ in $84 \%$ yield, but were difficult to separate from each other (Scheme 3 ).

A mixture of $\mathbf{2 3}$ and $\mathbf{2 4}$ was treated with dibutyltin 


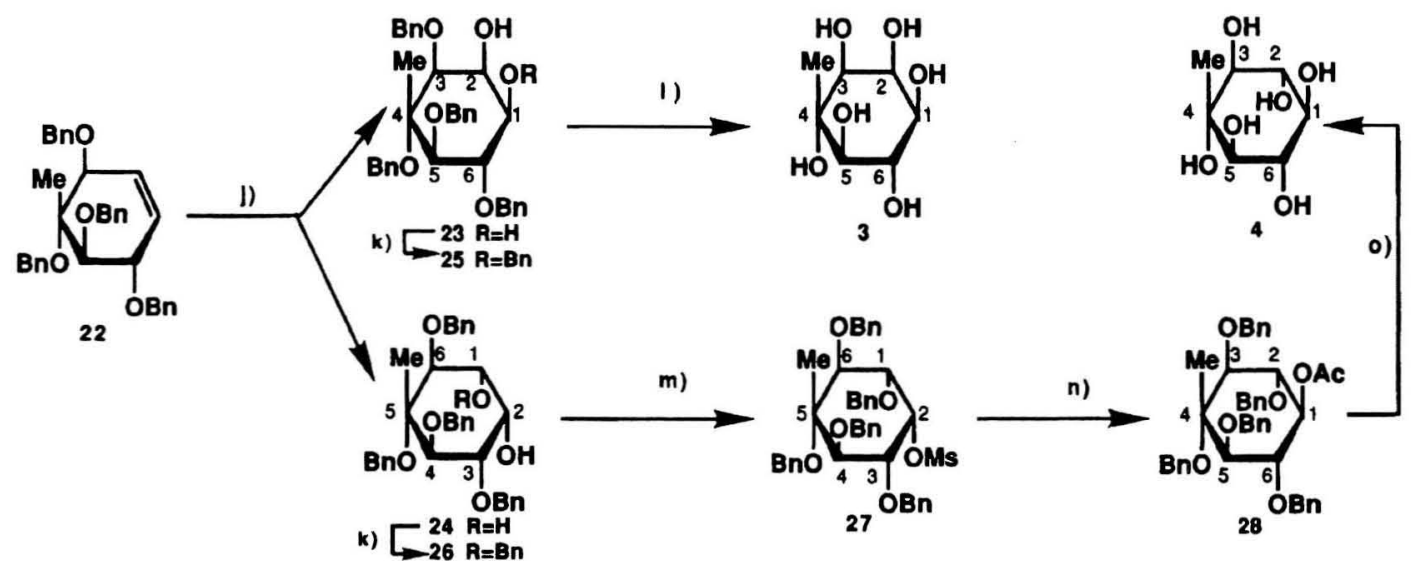

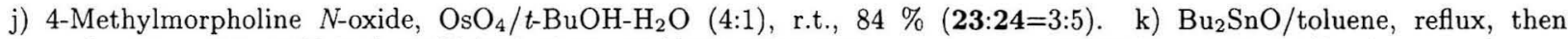
$\mathrm{BnBr} / \mathrm{DMF}, 80^{\circ} \mathrm{C}, 92 \%(\mathbf{2 5}), 74 \%$ (26). l) $\mathrm{H}_{2}, 10 \% \mathrm{Pd}-\mathrm{C} / \mathrm{MeOH}$, r.t., quant. m) MsCl$/ \mathrm{Py} ., 0{ }^{\circ} \mathrm{C}, 97 \%$. n) $\mathrm{CsOAc} / \mathrm{DMF}$, $80{ }^{\circ} \mathrm{C}, 93 \%$. o) $\mathrm{NaOMe} / \mathrm{EtOH}$, r.t., then $\mathrm{H}_{2}, 10 \% \mathrm{Pd}-\mathrm{C} / \mathrm{MeOH}$, r.t., quant.

Scheme 3

oxide and benzyl bromide to obtain selective benzylated (at equatorial hydroxyl group) products (25 and 26 ) in 92 and $74 \%$ yields, respectively. Debenzylation of $\mathbf{2 5}$ gave laminitol $\mathbf{3}$ in quantitative yield. Compound $\mathbf{3}$ was acetylated to purify and identify, because it seemed to be difficult to separate on a column of silica gel and to analyze the NMR data sufficiently in the free hydroxyl derivative 3 . Since the physical data of hexaacetate of 3 was completely identical with that reported for hexaacetate of laminitol, ${ }^{18}$ ) the configurations of $\mathbf{2 1}, \mathbf{2 3}$, and $\mathbf{2 4}$ were determined, respectively.

On the other hand, methylsulfonylation of $\mathbf{2 6}$ gave the corresponding methanesulfonate (27) in $97 \%$ yield. An $\mathrm{S}_{\mathrm{N}} 2$ reaction of $\mathbf{2 7}$ with cesium acetate gave the corresponding acetate derivative (28) in $93 \%$ yield. Deprotections of benzyl and acetyl groups of $\mathbf{2 8}$ gave mytilitol 4 in quantitative yield. Compound 4 was also acetylated in order to purify and identify for a similar reason as mentioned regarding compound $\mathbf{3}$. The physical data concerning the hexaacetate of 4 was identical with that reported for the hexaacetate of mytilitol. ${ }^{19)}$ In a similar manner as mentioned above, 25 may also be useful for the synthesis of 4 .

In conclusion, we clarified that $C$-methyl-branched 6-deoxyhex-5-enopyranoside derivatives are useful for the syntheses of $C$-methyl-branched inositol derivatives which are similar to inositol derivatives from 6-deoxyhex-5-enopyranosides derivatives.

\section{Experimental}

General Methods. All of the melting points were measured using a Yanaco MP-J3 apparatus and are uncorrected. The solutions were evaporated under reduced pressure at a bath temperature not exceeding $40{ }^{\circ} \mathrm{C}$. The optical rotations were measured in a $0.5 \mathrm{dm}$ tube with a JASCO DIP-140 polarimeter. The ${ }^{1} \mathrm{H}$ NMR spectra were recorded in chloroform- $d$ with JEOL PS-100, EX-90, and FX-200 spectrometers. IR spectra were recorded with Hitachi 270-
30 spectrometers. Elemental analyses were performed on a Perkin-Elmer $240 \mathrm{C}$ elemental analyzer. The chemical shifts, coupling constants, and IR frequencies were recorded in $\delta$, $\mathrm{Hz}$, and $\mathrm{cm}^{-1}$ units, respectively. Column chromatography was performed on silica gel (Silica gel 60, 70-230 mesh, Merck). Thin-layer chromatography (TLC) on Silica gel $60 \mathrm{~F}_{254}$ (Merck) was used to monitor the reactions and to certify the purity of the reaction products.

Methyl 6-Deoxy-3,4-bis ( $O$-methoxymethyl)-2- $O$ ( $p$-tolylsulfonyl)- $\alpha$-D- $x y l o-h e x-5$-enopyranoside (6). A mixture of methyl 3,4-bis $(O$-methoxymethyl)-2,6-bis[ $O$ ( $p$-tolylsulfonyl)]- $\alpha$-D-glucopyranoside (5) $(4.0 \mathrm{~g}, 6.8 \mathrm{mmol})$, $\mathrm{NaI}(5.1 \mathrm{~g}, 34 \mathrm{mmol}), \mathrm{Bu}_{4} \mathrm{NI}(1.3 \mathrm{~g}, 3.5 \mathrm{mmol})$, and molecular sieves $4 \mathrm{~A}(300 \mathrm{mg})$ in DMSO $\left(80 \mathrm{~cm}^{3}\right)$ was kept at $90{ }^{\circ} \mathrm{C}$ until the disappearance of 5 (for $14 \mathrm{~h}$ ). Then, DBU $(1.2 \mathrm{~g}, 7.9 \mathrm{mmol})$ was added to the reaction mixture, and they were kept for $6 \mathrm{~h}$. The reaction mixture was poured into a saturated ammonium chloride solution and extracted with ethyl acetate several times. The combined extract was washed, dried, and evaporated to give the corresponding 6deoxyhex-5-enopyranoside, which was purified on a column of silica gel (hexane/ethyl acetate=5/1), giving $1.8 \mathrm{~g}(63 \%)$ of 6 . We did not measure the optical rotation or analytical data, because compound 6 seems to be unstable to drying up and keeping. 6: Syrup; IR $1668 \mathrm{~cm}^{-1}(\mathrm{C}=\mathrm{C}) ;{ }^{1} \mathrm{H}$ NMR $\delta=7.85-7.34(\mathrm{~m}, 4 \mathrm{H}, \mathrm{Ph}), 4.84-4.64(\mathrm{~m}, 7 \mathrm{H}, \mathrm{H}-1, \mathrm{H}-6, \mathrm{H}-$ $6^{\prime}$, and $\left.-\mathrm{CH}_{2}-\times 2\right), 4.47$ (dd, $1 \mathrm{H}, J_{2,1}=3.4 \mathrm{~Hz}, J_{2,3}=9.5 \mathrm{~Hz}$, $\mathrm{H}-2), 4.00-3.92(\mathrm{~m}, 2 \mathrm{H}, \mathrm{H}-3$ and $\mathrm{H}-4), 3.43,3.34$, and 3.28 (each s, $3 \mathrm{H} \times 3, \mathrm{OMe} \times 3), 2.46(\mathrm{~s}, 3 \mathrm{H}, \mathrm{Me}-\mathrm{Ph})$.

Starting compound $\mathbf{5}$ was prepared as follows: To a mixture of methyl $\alpha$-D-glucopyranoside ( $15 \mathrm{~g}, 77 \mathrm{mmol}$ ) and pyridine $\left(75 \mathrm{~cm}^{3}\right)$ in dichloromethane $\left(150 \mathrm{~cm}^{3}\right), p$-toluenesulfonyl chloride ( $40 \mathrm{~g}, 0.21 \mathrm{~mol}, 2.7$ equiv) was carefully added in small portions at $-15^{\circ} \mathrm{C}$; the reaction mixture was kept for an additional $24 \mathrm{~h}$. After the disappearance of the starting material, the reaction mixture was poured into icewater, extracted with chloroform, washed with water, dried, and evaporated to give a syrupy product, which was purified on a column of silica gel (hexane/ethyl acetate $=2 / 1$ ), giving $31 \mathrm{~g}(80 \%)$ of the corresponding 2,6-ditosylate derivative. The structure was identified by NMR data concerning the 
3,4-di- $O$-acetyl derivative, methyl 3,4-di- $O$-acety]-2,6-bis $[O$ ( $p$-tolylsulfonyl)]- $\alpha$-D-glucopyranoside: [ ${ }^{1} \mathrm{H}$ NMR (FX-200) $\delta=7.89-7.14(\mathrm{~m}, 4 \mathrm{H} \times 2, \mathrm{Ph} \times 2), 5.37\left(\mathrm{dd}, 1 \mathrm{H}, J_{3,2}=9.9 \mathrm{~Hz}\right.$, $\left.J_{3,4}=9.5 \mathrm{~Hz}, \mathrm{H}-3\right), 4.88\left(\mathrm{dd}, 1 \mathrm{H}, J_{4,5}=9.5 \mathrm{~Hz}, \mathrm{H}-4\right), 4.80(\mathrm{~d}$, $\left.1 \mathrm{H}, J_{1,2}=3.5 \mathrm{~Hz}, \mathrm{H}-1\right), 4.36$ (dd, $\left.1 \mathrm{H}, \mathrm{H}-2\right), 4.15-3.87$ (m, $3 \mathrm{H}, \mathrm{H}-5, \mathrm{H}-6$, and $\left.\mathrm{H}-6^{\prime}\right), 3.32$ ( $\left.\mathrm{s}, 3 \mathrm{H}, \mathrm{OMe}\right), 2.43$ and 2.35 (each $\mathrm{s}, 3 \mathrm{H} \times 2, \mathrm{Me}-\mathrm{Ph} \times 2$ ), 1.91 and 1.73 (each s, $3 \mathrm{H} \times 2$, $\mathrm{OAc} \times 2)$ ]. To a chloroform solution of the above-mentioned methyl 2,6-bis $[O$-( $p$-tolylsulfonyl) $]-\alpha$-D-glucopyranoside (20 $\mathrm{g}, 40 \mathrm{mmol}$ ) and an excess amount of dimethoxymethane, a suitable amount of $\mathrm{P}_{2} \mathrm{O}_{5}$ was added and stirred for 12 h at r.t. until disappearance of the starting material. The reaction mixture was filtered through a celite bed; the filtrate was first washed with aq $\mathrm{NaHCO}_{3}$, then with water, dried, and evaporated to give $23 \mathrm{~g}(97 \%)$ of a pure residue 5 (on TLC). 5: $[\alpha]_{D}^{25}+69^{\circ}\left(c 0.80, \mathrm{CHCl}_{3}\right) ;{ }^{1} \mathrm{H}$ NMR (FX200) $\delta=7.81-7.25(\mathrm{~m}, 4 \mathrm{H} \times 2, \mathrm{Ph} \times 2), 4.80-4.55(\mathrm{~m}, 2 \mathrm{H} \times 2$, $\left.-\mathrm{CH}_{2}-\times 2\right), 4.34\left(\mathrm{dd}, 1 \mathrm{H}, \mathrm{J}_{6,5}=2.0 \mathrm{~Hz}, \mathrm{H}-6\right), 4.30(\mathrm{~d}, 1 \mathrm{H}$, $J_{1,2}=3.7 \mathrm{~Hz}, \mathrm{H}-1$ ), 4.25 (dd, $1 \mathrm{H}, J_{2,3}=9.8 \mathrm{~Hz}, \mathrm{H}-2$ ), 4.11 (dd, $1 \mathrm{H}, J_{6^{\prime}, 5}=6.1 \mathrm{~Hz}, J_{6^{\prime}, 6}=11.0 \mathrm{~Hz}, \mathrm{H}-6^{\prime}$ ), 3.93 (dd, $1 \mathrm{H}$, $J_{3,4}=9.0 \mathrm{~Hz}, \mathrm{H}-3$ ), 3.75 (ddd, $1 \mathrm{H}, J_{5,4}=9.0 \mathrm{~Hz}, \mathrm{H}-5$ ), 3.39 (dd, $1 \mathrm{H}, \mathrm{H}-4$ ), 3.31, 3.27, and 3.18 (each s, $3 \mathrm{H} \times 3$, $\mathrm{OMe} \times 3$ ), $2.45(\mathrm{~s}, 3 \mathrm{H} \times 2, \mathrm{Me}-\mathrm{Ph} \times 2)$.

Methyl 6-Deoxy-3,4-bis( $O$-methoxymethyl)- $\alpha$-Dxylo-hex-5-enopyranoside (7). A mixture of 6 (7.0 g, $17 \mathrm{mmol})$ and sodium methoxide in methanol $(\mathrm{pH} 10)$ was refluxed for $14 \mathrm{~h}$ until the disappearance of 6 ; brine was then added and most of methanol was evaporated in order to extract with ethyl acetate. The extract was washed with water, dried, and evaporated to give $4.0 \mathrm{~g}(90 \%)$ of a syrupy 7, which was purified on a column of silica gel. 7: Syrup; $[\alpha]_{D}^{25}+126^{\circ}\left(c \quad 1.5, \mathrm{CHCl}_{3}\right)$; IR $1665 \mathrm{~cm}^{-1}(\mathrm{C}=\mathrm{C}), 1413$ and $1215 \mathrm{~cm}^{-1}(\mathrm{~S}=\mathrm{O}) ;{ }^{1} \mathrm{H}$ NMR $(\mathrm{FX}-200) \delta=4.86-4.47(\mathrm{~m}, 7 \mathrm{H}$, $\left.\mathrm{H}-1, \mathrm{H}-6, \mathrm{H}-6^{\prime},-\mathrm{CH}_{2}-\times 2\right), 4.04$ (dd, $1 \mathrm{H}, J_{4,3}=8.5 \mathrm{~Hz}, J_{4,6}=$ $1.7 \mathrm{~Hz}, \mathrm{H}-4), 3.84-3.61$ ( $\mathrm{m}, 3 \mathrm{H}, \mathrm{H}-2, \mathrm{H}-3$, and $\mathrm{OH}), 3.48$, 3.46 , and 3.45 (each s, $3 \mathrm{H} \times 3, \mathrm{OMe} \times 3$ ). Found: C, 50.26; $\mathrm{H}, 7.39 \%$. Calcd for $\mathrm{C}_{11} \mathrm{H}_{20} \mathrm{O}_{7}: \mathrm{C}, 49.99 ; \mathrm{H}, 7.63 \%$.

Methyl 2- $O$-Benzyl-6-deoxy-3,4-bis $(O$-methoxymethyl)- $\alpha$-D- $x y l a-h e x-5$-enopyranoside (8). To a solution of $7(6.4 \mathrm{~g}, 24 \mathrm{mmol})$ in DMF $\left(100 \mathrm{~cm}^{3}\right)$, sodium hydride ( $55 \%$ in oil, $1.6 \mathrm{~g}, 37 \mathrm{mmol}$ ) was added at r.t. and stirred for $30 \mathrm{~min}$. Then benzyl bromide $(6.2 \mathrm{~g}, 36 \mathrm{mmol})$ was added to the reaction mixture. The usual work up of the reaction mixture gave $8.2 \mathrm{~g}(97 \%)$ of the corresponding 2- $O$-benzyl derivative 8 , which was purified on a column of silica gel (hexane/ethyl acetate $=5 / 1$ ). 8: Syrup; $[\alpha]_{\mathrm{D}}^{25}+16^{\circ}\left(\right.$ c $\left.1.0, \mathrm{CHCl}_{3}\right)$; IR $1668 \mathrm{~cm}^{-1}(\mathrm{C}=\mathrm{C}) ;{ }^{1} \mathrm{H}$ NMR $(\mathrm{FX}-200) \delta=7.39-7.30(\mathrm{~m}, 5 \mathrm{H}, \mathrm{Ph}), 4.92-4.75(\mathrm{~m}, 2 \mathrm{H} \times 3$, $\left.-\mathrm{CH}_{2}-\times 3\right), 4.69$ and $4.66\left(\mathrm{~s} \times 2,1 \mathrm{H} \times 2, \mathrm{H}-6\right.$ and $\left.\mathrm{H}-6^{\prime}\right), 4.59$ (d, $1 \mathrm{H}, J_{1,2}=3.7 \mathrm{~Hz}, \mathrm{H}-1$ ) 4.01 (ddd, $1 \mathrm{H}, J_{4,2}=1.7 \mathrm{~Hz}$, $\left.J_{4,3}=9.3 \mathrm{~Hz}, \mathrm{H}-4\right), 3.95$ (dd, $1 \mathrm{H}, J_{3,2}=9.3 \mathrm{~Hz}, \mathrm{H}-3$ ), 3.52 (ddd, $1 \mathrm{H}, \mathrm{H}-2$ ), 3.46, 3.44, and 3.39 (each s, $3 \mathrm{H} \times 3, \mathrm{OMe} \times 3$ ). Found: C, $60.90 ; \mathrm{H}, 7.45 \%$. Calcd for $\mathrm{C}_{18} \mathrm{H}_{26} \mathrm{O}_{7}$ : C, 61.00 ; $\mathrm{H}, 7.40 \%$.

2L-(2,4/3)-4-O-Benzyl-2,3-bis ( $O$-methoxymethyl)2,3,4-trihydroxy-5-cyclohexen-1-one (9). A mixture of $8(2.9 \mathrm{~g}, 8.3 \mathrm{mmol})$ and mercury(II) acetate $(3.4 \mathrm{~g}, 11 \mathrm{mmol})$ in a solution [acetone $\left(175 \mathrm{~cm}^{3}\right)$, water $\left(70 \mathrm{~cm}^{3}\right)$, and acetic acid $\left(2.5 \mathrm{~cm}^{3}\right)$ ] was stirred at the reflux temperature for 5 h. The reaction mixture was evaporated, dissolved in chloroform, washed with water, dried, and again evaporated to give the corresponding cyclohexanone derivative, which was used for the next reaction without purification. Then, the above-mentioned product was treated with acetic anhydride in pyridine at r.t. for $12 \mathrm{~h}$, and evaporated to give $2.1 \mathrm{~g}$ ( $77 \%, 2$ steps) of the enone derivative $\mathbf{9}$, which was purified on a column of silica gel (hexane/ethyl acetate $=3 / 1$ ). 9: Syrup; $[\alpha]_{\mathrm{D}}^{25}+111^{\circ}\left(\right.$ c $\left.2.1, \mathrm{CHCl}_{3}\right)$; IR $1701 \mathrm{~cm}^{-1}(\mathrm{C}=\mathrm{O})$; ${ }^{1} \mathrm{H}$ NMR (FX-200) $\delta=7.39-7.31(\mathrm{~m}, 5 \mathrm{H}, \mathrm{Ph}), 6.83(\mathrm{dd}, 1 \mathrm{H}$, $\left.J_{6,4}=2.0 \mathrm{~Hz}, J_{6,5}=10.0 \mathrm{~Hz}, \mathrm{H}-6\right), 6.04$ (dd, $1 \mathrm{H}, \mathrm{H}-5$ ), $4.98-$ $4.77\left(\mathrm{~m}, 2 \mathrm{H} \times 3,-\mathrm{CH}_{2}-\times 3\right), 4.36$ (ddd, $1 \mathrm{H}, J_{4,3}=8.3 \mathrm{~Hz}, \mathrm{H}-$ 4), $4.22\left(\mathrm{~d}, 1 \mathrm{H}, J_{2,3}=11.0 \mathrm{~Hz}, \mathrm{H}-2\right), 4.06$ (dd, $\left.1 \mathrm{H}, \mathrm{H}-3\right)$, 3.49 and 3.43 (each s, $3 \mathrm{H} \times 2, \mathrm{OMe} \times 2$ ). Found: $\mathrm{C}, 63.58 ; \mathrm{H}$, $6.86 \%$. Calcd for $\mathrm{C}_{17} \mathrm{H}_{22} \mathrm{O}_{6}$ : C, $63.34 ; \mathrm{H}, 6.88 \%$.

1D- $(1,3 / 2,4)-1,4-\mathrm{Di}-O$-benzyl-2,3-bis $(O$-methoxymethyl)-5-cyclohexene-1,2,3,4-tetrol (10). To a mixture of $9(1.3 \mathrm{~g}, 3.9 \mathrm{mmol})$ and cerium(III) chloride $\left[\mathrm{CeCl}_{3} \cdot 7 \mathrm{H}_{2} \mathrm{O}(1.8 \mathrm{~g}, 4.7 \mathrm{mmol})\right]$ in a mixed solvent of ethanol and dichloromethane $(2: 1)$ was added an ethanolic solution $\left(9.0 \mathrm{~cm}^{3}\right)$ of sodium tetrahydroborate $(0.18 \mathrm{~g}, 4.7 \mathrm{mmol})$ at $-78^{\circ} \mathrm{C}$ under argon. After $30 \mathrm{~min}$, the temperature was raised to room temperature; the reaction mixture was then filtered through a celite bed, and the filtrate was evaporated to give a residue. The residue was dissolved in chloroform, washed with water, dried over anhydrous magnesium sulfate, and evaporated to give the corresponding hydroxy derivative. The above-mentioned product was treated with sodium hydride [0.23 $\mathrm{g}$ ( $55 \%$ in oil), $5.3 \mathrm{mmol}]$ and benzyl bromide $(0.92 \mathrm{~g}, 5.4 \mathrm{mmol})$ in DMF $\left(2.5 \mathrm{~cm}^{3}\right)$ in a similar manner as described regarding the synthesis of 8 , to give $1.45 \mathrm{~g}(89 \%)$ of 10, which was purified on a column of silica gel (hexane/ethyl acetate=4/1). 10: Syrup; $[\alpha]_{\mathrm{D}}^{18}+165^{\circ}\left(\right.$ c $\left.0.79, \mathrm{CHCl}_{3}\right)$; ${ }^{1} \mathrm{H} N M R(\mathrm{FX}-200) \delta=7.35-7.27(\mathrm{~m}, 5 \mathrm{H} \times 2, \mathrm{Ph} \times 2), 5.73$ (s, 2H, H-5 and $\mathrm{H}-6), 4.90$ (s, $\left.2 \mathrm{H} \times 2,-\mathrm{CH}_{2}-\times 2\right), 4.65(\mathrm{ABq}$, $\left.2 \mathrm{H} \times 2,-\mathrm{CH}_{2}-\times 2\right), 4.17$ and 3.79 [each dd $\left(\mathrm{A}_{2} \mathrm{~B}_{2}\right), 4 \mathrm{H}, J=5.1$ and $2.4 \mathrm{~Hz}, \mathrm{H}-1, \mathrm{H}-4$ and $\mathrm{H}-2, \mathrm{H}-3], 3.42(\mathrm{~s}, 3 \mathrm{H} \times 2, \mathrm{OMe} \times 2)$. Found: $\mathrm{C}, 69.29 ; \mathrm{H}, 7.39 \%$. Calcd for $\mathrm{C}_{24} \mathrm{H}_{30} \mathrm{O}_{6}$ : C, 69.54; $\mathrm{H}, 7.30 \%$.

1D-3,6-Di- $O$-benzyl-4,5-bis $(O$-methoxymethyl)-1, 2,3,5/4,6-cyclohexanehexol (11). To the solution of $10(90 \mathrm{mg}, 0.22 \mathrm{mmol})$ in $1.5 \mathrm{~cm}^{3}$ of a mixed solvent (acetone $/ \mathrm{H}_{2} \mathrm{O}=4 / 1$ ) was added 4-methylmorpholine $\mathrm{N}$-oxide (31 $\mathrm{mg}, 0.26 \mathrm{mmol}$ ) and osmium tetraoxide in 2-methyl-2propanol $\left(0.05 \mathrm{~mol} \mathrm{dm}^{-3}: 0.1 \mathrm{~cm}^{3}, 0.005 \mathrm{mmol}\right)$ and stirred for $12 \mathrm{~h}$. To the reaction mixture, sodium hydrogensulfite (54 $\mathrm{mg}, 0.52 \mathrm{mmol}$ ) was added and stirred for $1 \mathrm{~h}$; the reaction mixture was then filtered, evaporated, and extracted with chloroform. The extract was washed with water, dried over anhydrous magnesium sulfate, and evaporated to give 11, which was purified on a column of silica gel (hexane/eth$\mathrm{yl}$ acetate $=1 / 1$ ). The yield of 11 was $82 \mathrm{mg}(83 \%)$. 11: $\mathrm{Mp} 113-114^{\circ} \mathrm{C}$ (recrystallized from hexane-ethyl acetate); $[\alpha]_{\mathrm{D}}^{18}-3.9^{\circ}$ ( c $\left.0.70, \mathrm{CHCl}_{3}\right)$; IR $3430 \mathrm{~cm}^{-1}(\mathrm{O}-\mathrm{H}) ;{ }^{1} \mathrm{H}$ NMR $(\mathrm{FX}-200) \delta=7.35-7.26(\mathrm{~m}, 5 \mathrm{H} \times 2, \mathrm{Ph} \times 2), 4.95-4.67(\mathrm{~m}$, $\left.2 \mathrm{H} \times 4,-\mathrm{CH}_{2}-\times 4\right), 4.16\left(\mathrm{dd}, 1 \mathrm{H}, J_{2,1}=J_{2,3}=2.7 \mathrm{~Hz}, \mathrm{H}-2\right)$, 3.95 (dd, $\left.1 \mathrm{H}, J_{4,3}=9.8 \mathrm{~Hz}, J_{4,5}=9.0 \mathrm{~Hz}, \mathrm{H}-4\right), 3.76$ (dd, $1 \mathrm{H}$, $\left.J_{6,5}=9.5 \mathrm{~Hz}, J_{6,1}=9.3 \mathrm{~Hz}, \mathrm{H}-6\right), 3.52-3.46(\mathrm{~m}, 1 \mathrm{H}, \mathrm{H}-1)$, 3.49 (dd, $1 \mathrm{H}, \mathrm{H}-5$ ), 3.43 (each s, $3 \mathrm{H} \times 2$, OMe $\times 2$ ), 3.37 (dd, $1 \mathrm{H}, \mathrm{H}-3), 2.47(\mathrm{~s}, 1 \mathrm{H}, \mathrm{OH}), 2.36\left(\mathrm{~d}, 1 \mathrm{H}, \mathrm{J}_{\mathrm{OH}, 1}=4.9 \mathrm{~Hz}, \mathrm{OH}\right)$. Found: $\mathrm{C}, 64.06 ; \mathrm{H}, 7.11 \%$. Calcd for $\mathrm{C}_{24} \mathrm{H}_{32} \mathrm{O}_{8}$ : C, 64.27; $\mathrm{H}, 7.19 \%$.

1D-3,6-Di- $O$-benzyl-1,4,5-tris( $O$-methoxymethyl)1,2,3,5/4,6-cyclohexanehexol (12). A mixture of cisdiol derivative $11(73 \mathrm{mg}, 0.16 \mathrm{mmol})$ and dibutyltin oxide 
( $53 \mathrm{mg}, 0.21 \mathrm{mmol}, 1.3$ equiv) in benzene $\left(2.0 \mathrm{~cm}^{3}\right)$ was refluxed for $9 \mathrm{~h}$; triethylamine $(0.15 \mathrm{~g}, 1.4 \mathrm{mmol}, 8.9$ equiv) and chloromethyl methyl ether (92 $\mathrm{mg}, 1.1 \mathrm{mmol}, 7.1$ equiv) were then added to the reaction mixture at r.t. and kept until the disappearance of $\mathbf{1 1}$ (for $30 \mathrm{~min}$ ). To the reaction mixture, potassium fluoride solution was added and the precipitate was filtered off. After separating the benzene layer, the mother solution was extracted with ethyl acetate. The combined organic layer was dried over anhydrous magnesium sulfate and evaporated to give a residue, which was purified on a column of silica gel (ethyl acetate/hexane $=1 / 4$ ), giving $65 \mathrm{mg}(82 \%)$ of 12.12 : $\mathrm{Mp} 52-53^{\circ} \mathrm{C}$ (recrystallized from ether-hexane); $[\alpha]_{\mathrm{D}}^{18}+32^{\circ}\left(c 1.0, \mathrm{CHCl}_{3}\right)$; IR 3478 $\mathrm{cm}^{-1}(\mathrm{O}-\mathrm{H}) ;^{1} \mathrm{H}$ NMR (FX-200) $\delta=7.38-7.26(\mathrm{~m}, 5 \mathrm{H} \times 2$, $\mathrm{Ph} \times 2), 4.92-4.62\left(\mathrm{~m}, 2 \mathrm{H} \times 5,-\mathrm{CH}_{2}-\times 5\right), 4.18(\mathrm{dd}, 1 \mathrm{H}$, $\left.J_{2,1}=J_{2,3}=3.3 \mathrm{~Hz}, \mathrm{H}-2\right), 3.96\left(\mathrm{dd}, 1 \mathrm{H}, J_{4,5}=9.3 \mathrm{~Hz}, J_{4,3}=9.8\right.$ $\mathrm{Hz}, \mathrm{H}-4), 3.89$ (dd, $1 \mathrm{H}, J_{6,5}=J_{6,1}=9.8 \mathrm{~Hz}, \mathrm{H}-6$ ), $3.42-3.33$ $(\mathrm{m}, 2 \mathrm{H}, \mathrm{H}-3$ and $\mathrm{H}-5), 3.42,3.39$, and 3.35 (each s, $3 \mathrm{H} \times 3$, $\mathrm{OMe} \times 3), 2.47(\mathrm{~s}, 1 \mathrm{H}, \mathrm{OH})$. Found: $\mathrm{C}, 63.67 ; \mathrm{H}, 7.21 \%$. Calcd for $\mathrm{C}_{26} \mathrm{H}_{36} \mathrm{O}_{9}$ : C, 63.40; $\mathrm{H}, 7.37 \%$.

1D-2,3,6-Tri- $O$-benzyl-1,4,5-tris $(O$-methoxymethyl)-1,2,3,5/4,6-cyclohexanehexol (13). Benzylation of $12(65 \mathrm{mg}, 0.13 \mathrm{mmol})$ with sodium hydride [9.0 $\mathrm{mg}(55 \%$ in oil), $0.21 \mathrm{mmol}, 1.6$ equiv] and benzyl bromide ( $35 \mathrm{mg}, 0.20$ mmol, 1.5 equiv) in a similar reaction from 7 to 8 gave the corresponding tri- $O$-benzylated derivative, which was purified on a column of silica gel (hexane/ethyl acetate $=4 / 1$ ), giving $73 \mathrm{mg}$ (96\%) of 13. 13: $\mathrm{Mp} 70-72{ }^{\circ} \mathrm{C}$ (recrystallized from ethanol-hexane); $[\alpha]_{\mathrm{D}}^{18}+8.6^{\circ}\left(\mathrm{c} 0.30, \mathrm{CHCl}_{3}\right) ;{ }^{1} \mathrm{H}$ NMR $(\mathrm{FX}-200) \delta=7.38-7.27(\mathrm{~m}, 5 \mathrm{H} \times 3, \mathrm{Ph} \times 3), 4.94-4.58(\mathrm{~m}$, $\left.2 \mathrm{H} \times 6,-\mathrm{CH}_{2}-\times 6\right), 4.07\left(\mathrm{dd}, 1 \mathrm{H}, J_{4,3}=J_{4,5}=9.5 \mathrm{~Hz}, \mathrm{H}-4\right.$ ), $3.96\left(\mathrm{dd}, 1 \mathrm{H}, J_{2,1}=2.4 \mathrm{~Hz}, J_{2,3}=2.0 \mathrm{~Hz}, \mathrm{H}-2\right), 3.94(\mathrm{dd}, 1 \mathrm{H}$, $\left.J_{6,1}=J_{6,5}=9.8 \mathrm{~Hz}, \mathrm{H}-6\right), 3.48$ (dd, $\left.1 \mathrm{H}, \mathrm{H}-1\right), 3.47$ (dd, $1 \mathrm{H}$, $\mathrm{H}-5$ ), 3.35 (dd, $1 \mathrm{H}, \mathrm{H}-3$ ), 3.41, 3.39, and 3.30 (each s, $3 \mathrm{H} \times 3$, $\mathrm{OMe} \times 3$ ). Found: $\mathrm{C}, 68.35 ; \mathrm{H}, 7.27 \%$. Calcd for $\mathrm{C}_{33} \mathrm{H}_{42} \mathrm{O}_{9}$ : $\mathrm{C}, 68.02 ; \mathrm{H}, 7.27 \%$.

1D- 2,3,6-Tri- $O$-benzyl- $1,2,3,5 / 4,6$ - cyclohexanehexol (2,3,6-Tri-O-benzyl-D-myo-inositol) (2). Compound 13 (50 $\mathrm{mg}, 0.086 \mathrm{mmol}$ ) was treated with a $0.1 \mathrm{M}$ hydrogen chloride methanolic solution $\left(1.0 \mathrm{~cm}^{3}\right)$ at $60-$ $65{ }^{\circ} \mathrm{C}$ for $1 \mathrm{~h}\left(1 \mathrm{M}=1 \mathrm{~mol} \mathrm{dm}^{-3}\right)$ until the disappearance of 13; the solution was neutralized with an ion-exchange resin (Amberlist, A-26) and evaporated to give a residue, which was purified on a column of silica gel (hexane/ethyl acetate $=2 / 1)$, giving $35 \mathrm{mg}(90 \%)$ of 2. 2: Mp 117-119 ${ }^{\circ} \mathrm{C}$ (recrystallized from ethanol-water); $[\alpha]_{\mathrm{D}}^{24}+12^{\circ}$ (c 0.79 , $\left.\mathrm{CHCl}_{3}\right)\left[\mathrm{lit},{ }^{16)} \mathrm{Mp} 117-119^{\circ} \mathrm{C} ;[\alpha]_{\mathrm{D}}^{16}+15.5^{\circ}\left(\mathrm{CHCl}_{3}\right){ }^{\#}\right]$; IR $3436 \mathrm{~cm}^{-1}(\mathrm{O}-\mathrm{H}) ;{ }^{1} \mathrm{H}$ NMR (FX-200) $\delta=7.40-7.29(\mathrm{~m}$, $5 \mathrm{H} \times 3, \mathrm{Ph} \times 3), 4.97-4.55\left(\mathrm{~m}, 2 \mathrm{H} \times 3,-\mathrm{CH}_{2}-\times 3\right), 4.08$ (dd, $\left.1 \mathrm{H}, J_{2,1}=J_{2,3}=2.7 \mathrm{~Hz}, \mathrm{H}-2\right), 4.01\left(\mathrm{ddd}, 1 \mathrm{H}, J_{4,3}=J_{4,5}=9.8\right.$ $\left.\mathrm{Hz}, J_{4, \mathrm{OH}}=2.7 \mathrm{~Hz}, \mathrm{H}-4\right), 3.68\left(\mathrm{dd}, 1 \mathrm{H}, J_{6,5}=J_{6,1}=9.2 \mathrm{~Hz}\right.$, $\mathrm{H}-6), 3.52$ (ddd, $\left.1 \mathrm{H}, J_{1, \mathrm{OH}}=6.6 \mathrm{~Hz}, \mathrm{H}-1\right), 3.47$ (ddd, $1 \mathrm{H}$, $\left.J_{5, \mathrm{OH}}=2.7 \mathrm{~Hz}, \mathrm{H}-5\right), 3.27$ (dd, $\left.1 \mathrm{H}, \mathrm{H}-3\right), 2.65$ and 2.61 (each s, $1 \mathrm{H} \times 2, \mathrm{OH} \times 2), 2.34(\mathrm{~d}, 1 \mathrm{H}, \mathrm{OH})$. Found: C, $72.06 ; \mathrm{H}$, 6.55\%. Calcd for $\mathrm{C}_{27} \mathrm{H}_{30} \mathrm{O}_{6}$ : C, $71.98 ; \mathrm{H}, 6.71 \%$.

Methyl 2,3-Di- $O$-benzyl-4- $C$-methyl-6- $O$-triphenylmethyl- $\alpha$-D-glucopyranoside (14). Compound 14 was prepared ${ }^{17)}$ from the corresponding 4-ulose (17 g, 27 $\mathrm{mmol}$ ) by treatment with methyllithium at $-78^{\circ} \mathrm{C}$, in $72 \%$ yield $(12 \mathrm{~g})$.

\#Concentration was not stated.
Methyl 2,3,4-Tri- $O$-benzyl-4- $C$-methyl-6- $O$-triphenylmethyl- $\alpha$-D-glucopyranoside (15). Benzylation of $14(0.72 \mathrm{~g}, 1.14 \mathrm{mmol})$ with sodium hydride [0.33 $\mathrm{g}$ ( $55 \%$ in oil), $7.6 \mathrm{mmol}, 6.6$ equiv] and benzyl bromide (1.4 $\mathrm{g}, 8.4 \mathrm{mmol}, 7.4$ equiv) in a similar manner as described regarding the synthesis of $\mathbf{8}$ from $\mathbf{7}$, gave $\mathbf{1 5}$, which was separated on a column of silica gel (hexane/ethyl acetate $=9 / 1$ ), in $78 \%$ yield $(0.64 \mathrm{~g})$. However, it was inevitable to retain a slight amount of contaminant. 15: ${ }^{1} \mathrm{H}$ NMR $\delta=7.70-7.19$ $(\mathrm{m}, 5 \mathrm{H} \times 6, \mathrm{Ph} \times 6), 4.72\left(\mathrm{~d}, 1 \mathrm{H}, J_{1,2}=3.4 \mathrm{~Hz}, \mathrm{H}-1\right), 4.16$ (dd, $\left.1 \mathrm{H}, J_{5,6}=1.0 \mathrm{~Hz}, J_{5,6^{\prime}}=8.1 \mathrm{~Hz}, \mathrm{H}-5\right), 4.08(\mathrm{~d}, 1 \mathrm{H}, \mathrm{H}-3), 3.51$ (dd, $\left.1 \mathrm{H}, J_{2,3}=9.8 \mathrm{~Hz}, \mathrm{H}-2\right), 3.39$ (dd, $1 \mathrm{H}, J_{6,6^{\prime}}=9.3 \mathrm{~Hz}, \mathrm{H}-$ 6), $3.23\left(\mathrm{dd}, 1 \mathrm{H}, \mathrm{H}-6^{\prime}\right), 3.13(\mathrm{~s}, 3 \mathrm{H}, \mathrm{OMe}), 1.12(\mathrm{~s}, 3 \mathrm{H}, \mathrm{C}-$ $\mathrm{Me})$.

Methyl 2,3,4-Tri- $O$-benzyl-4- $C$-methyl- $\alpha$-D-glucopyranoside (16). A solution of $15(0.50 \mathrm{~g}, 0.69 \mathrm{mmol})$ in $70 \%$ acetic acid $\left(7.5 \mathrm{~cm}^{3}\right)$ was stirred at $60{ }^{\circ} \mathrm{C}$ until the disappearance of $\mathbf{1 5}$; the solution was then evaporated to give 16, which was purified on a column of silica gel (hexane/ethyl acetate $=3 / 1$ ), in quantitative yield. 16: $\mathrm{Mp} 76^{\circ} \mathrm{C}$ (recrystallized from ethanol-hexane); $[\alpha]_{\mathrm{D}}^{25}+65^{\circ}$ (c 1.0, $\mathrm{CHCl}_{3}$ ); IR $3472 \mathrm{~cm}^{-1}$ (O-H); ${ }^{1} \mathrm{H}$ NMR (FX-200) $\delta=7.46-7.18(\mathrm{~m}, 5 \mathrm{H} \times 3, \mathrm{Ph} \times 3), 4.74\left(\mathrm{~d}, 1 \mathrm{H}, J_{1,2}=4.0 \mathrm{~Hz}\right.$, $\mathrm{H}-1), 4.16(\mathrm{~d}, 1 \mathrm{H}, \mathrm{H}-3), 4.00\left(\mathrm{dd}, 1 \mathrm{H}, J_{5,6}=2.0 \mathrm{~Hz}, J_{5,6^{\prime}}=8.2\right.$ $\mathrm{Hz}, \mathrm{H}-6), 3.90$ (dd, $\left.1 \mathrm{H}, J_{6,6^{\prime}}=8.0 \mathrm{~Hz}, \mathrm{H}-6^{\prime}\right), 3.70$ (dd, $1 \mathrm{H}$, $\mathrm{H}-5$ ), 3.58 (dd, $\left.1 \mathrm{H}, J_{2,3}=10.0 \mathrm{~Hz}, \mathrm{H}-2\right), 3.48$ (s, 3H, OMe), $1.42(\mathrm{~s}, 3 \mathrm{H}, \mathrm{C}-\mathrm{Me})$. Found: $\mathrm{C}, 72.76 ; \mathrm{H}, 7.25 \%$. Calcd for $\mathrm{C}_{29} \mathrm{H}_{34} \mathrm{O}_{6}: \mathrm{C}, 72.78 ; \mathrm{H}, 7.16 \%$.

Methyl 2,3,4-Tri- $O$-benzyl-4- $C$-methyl-6- $O$ - ( $p$-tolylsulfonyl)- $\alpha$-D-glucopyranoside (17). A mixture of $16(10 \mathrm{~g}, 21 \mathrm{mmol})$ and $p$-toluenesulfonyl chloride $(6.0 \mathrm{~g}, 31$ mmol, 1.5 equiv) in pyridine $\left(100 \mathrm{~cm}^{3}\right)$ was stirred at r.t. until the disappearance of $\mathbf{1 6}$ (for $3 \mathrm{~h}$ ). The reaction mixture was then poured into $1.0 \%$ hydrochloric acid, extracted with ethyl acetate, washed with water, dried over anhydrous magnesium sulfate, and evaporated to give the corresponding $p$-toluenesulfonate $\mathbf{1 7}$, which was purified on a column of silica gel (hexane/ethyl acetate $=7 / 1$ ), in $99 \%$ yield $(13 \mathrm{~g}$ ). 17: Syrup; $[\alpha]_{\mathrm{D}}^{25}+6.5^{\circ}\left(c 0.95, \mathrm{CHCl}_{3}\right)$; IR 1455 and 1176 $\mathrm{cm}^{-1}(\mathrm{~S}=\mathrm{O})$; $^{1} \mathrm{H}$ NMR $(\mathrm{FX}-200) \delta=7.53-7.18(\mathrm{~m}, 5 \mathrm{H} \times 3$, $4 \mathrm{H} \times 1, \mathrm{Ph} \times 4), 4.90\left(\mathrm{~d}, 1 \mathrm{H}, J_{1,2}=3.8 \mathrm{~Hz}, \mathrm{H}-1\right), 4.61(\mathrm{~s}, 2 \mathrm{H}$, $\left.-\mathrm{CH}_{2}-\right)$, 4.70-4.45 (ABq, $\left.2 \mathrm{H} \times 2,-\mathrm{CH}_{2}-\times 2\right), 4.08(\mathrm{~d}, 1 \mathrm{H}$, $\mathrm{H}-3$ ), 3.88 (dd, 1H, J $J_{2,3}=10.0 \mathrm{~Hz}, \mathrm{H}-2$ ), 3.38 (s, 3H, OMe), 2.40 (s, 3H, Me-Ph), 1.24 (s, 3H, C-Me). Found: C, 68.11; $\mathrm{H}, 6.53 \%$. Calcd for $\mathrm{C}_{36} \mathrm{H}_{40} \mathrm{O}_{8} \mathrm{~S}: \mathrm{C}, 68.34 ; \mathrm{H}, 6.37 \%$.

Methyl 2,3,4-Tri- $O$-benzyl-6-deoxy-4- $C$-methyl- $\alpha$ D-xylo-hex-5-enopyranoside (18). A mixture of 17 (3.1 $\mathrm{g}, 4.9 \mathrm{mmol}$ ), molecular sieves $4 \mathrm{~A}$ (10 pieces), sodium iodide ( $5.5 \mathrm{~g}, 37 \mathrm{mmol}, 7.5$ equiv), and tetrabutylammonium iodide (1.4 $\mathrm{g}, 3.6 \mathrm{mmol}, 0.73$ equiv) in DMSO $\left(45 \mathrm{~cm}^{3}\right)$ was stirred at $120{ }^{\circ} \mathrm{C}$ until the disappearance of 17 (for $12 \mathrm{~h}$ ). To the above reaction mixture, DBU ( $5.6 \mathrm{~g}, 37 \mathrm{mmol}, 7.5$ equiv) was added and stirred at $120{ }^{\circ} \mathrm{C}$ for $12 \mathrm{~h}$. The reaction mixture was filtered, and the filtrate was poured into water, extracted with ethyl acetate, washed with water, dried over anhydrous magnesium sulfate, and evaporated to give 6-deoxyhex-5-enopyranoside 18 , which was purified on a column of silica gel (hexane/ethyl acetate $=8 / 1)$, in $61 \%$ yield $(1.4$ g). 18: Syrup; $[\alpha]_{\mathrm{D}}^{25}-16^{\circ}\left(c 1.0, \mathrm{CHCl}_{3}\right)$; IR $1653 \mathrm{~cm}^{-1}$ $(\mathrm{C}=\mathrm{C}) ;{ }^{1} \mathrm{H}$ NMR $(\mathrm{FX}-200) \delta=7.52-7.10(\mathrm{~m}, 5 \mathrm{H} \times 3, \mathrm{Ph} \times 3)$, $4.67(\mathrm{~d}, 1 \mathrm{H}, \mathrm{H}-6), 4.65\left(\mathrm{~d}, 1 \mathrm{H}, J_{1,2}=3.4 \mathrm{~Hz}, \mathrm{H}-1\right), 4.65$ (d, $\left.1 \mathrm{H}, J_{6^{\prime}, 6}=2.0 \mathrm{~Hz}, \mathrm{H}-6^{\prime}\right), 3.65\left(\mathrm{dd}, 1 \mathrm{H}, J_{2,3}=9.8 \mathrm{~Hz}, \mathrm{H}-2\right)$, 
3.45 (s, 3H, OMe), 1.55 (s, 3H, C-Me). Found: C, 75.85; H, $7.13 \%$. Calcd for $\mathrm{C}_{29} \mathrm{H}_{32} \mathrm{O}_{5}: \mathrm{C}, 75.63 ; \mathrm{H}, 7.00 \%$.

2L-(2,4/3)-2,3,4-Tri- $O$-benzyl-2- $C$-methyl-2,3,4-trihydroxy-5-cyclohexen-1-one (20). A mixture of 18 (1.1 $\mathrm{g}, 2.4 \mathrm{mmol})$ and mercury(II) chloride $(2.8 \mathrm{~g}, 10 \mathrm{mmol}, 4.3$ equiv) in a mixed solvent (acetone/water $=2 / 5,27 \mathrm{~cm}^{3}$ ) was stirred at $80^{\circ} \mathrm{C}$ for $3 \mathrm{~h}$. The reaction mixture was filtered, and the filtrate was evaporated to remove most of the acetone. To the remaining solution, water was added and the solution was extracted with chloroform. The organic layer was washed with water, dried over anhydrous magnesium sulfate, and evaporated to give the corresponding epimeric cyclohexanone derivatives $(19 \alpha$ and $19 \beta)$, which were purified on a column of silica gel (hexane/ethyl acetate $=3 / 1)$ in a ratio of $1: 2(\alpha: \beta)$ in $72 \%$ yield $(0.77 \mathrm{~g}) .19 \alpha$ : IR $3452(\mathrm{O}$ $\mathrm{H})$ and $1730 \mathrm{~cm}^{-1}(\mathrm{C}=\mathrm{O}) ;{ }^{1} \mathrm{H}$ NMR $(\mathrm{FX}-200) \cdot \delta=7.48-7.16$ $(\mathrm{m}, 5 \mathrm{H} \times 3, \mathrm{Ph} \times 3), 4.88-4.55\left(\mathrm{ABq} \times 3,2 \mathrm{H} \times 3, \mathrm{PhCH}_{2}-\times 3\right)$, $4.30-4.20\left(\mathrm{~m}, 1 \mathrm{H}, J_{5,6}=4.2 \mathrm{~Hz}, \mathrm{H}-5\right), 3.86\left(\mathrm{~d}, 1 \mathrm{H}, J_{3.4}=7.6\right.$ $\mathrm{Hz}, \mathrm{H}-3$ ), 3.70 (dd, $\left.1 \mathrm{H}, J_{4,5}=7.8 \mathrm{~Hz}, \mathrm{H}-4\right), 2.75$ (dd, $1 \mathrm{H}, \mathrm{H}-$ 6), $2.65-2.50\left(\mathrm{~m}, 1 \mathrm{H}, J_{6,6^{\prime}}=15.4 \mathrm{~Hz}, \mathrm{H}-6^{\prime}\right), 2.55$ (bs, $1 \mathrm{H}$, $\mathrm{OH}), 1.57(\mathrm{~s}, 3 \mathrm{H}, \mathrm{C}-\mathrm{Me}) . \quad 19 \beta$ : IR $3452(\mathrm{O}-\mathrm{H})$ and 1730 $\mathrm{cm}^{-1}(\mathrm{C}=\mathrm{O}) ;{ }^{1} \mathrm{H}$ NMR (FX-200) $\delta=7.52-7.20(\mathrm{~m}, 5 \mathrm{H} \times 3$, $\mathrm{Ph} \times 3), 4.80\left(\mathrm{~s}, 2 \mathrm{H}, \mathrm{PhCH}_{2}-\right), 4.86-4.55(\mathrm{ABq} \times 2,2 \mathrm{H} \times 2$, $\left.\mathrm{PhCH}_{2}-\times 2\right), 4.26\left(\mathrm{~d}, 1 \mathrm{H}, J_{3,4}=8.5 \mathrm{~Hz}, \mathrm{H}-3\right), 3.90-3.84(\mathrm{~m}$, $1 \mathrm{H}, \mathrm{H}-5$ ), 3.79 (dd, $1 \mathrm{H}, J_{4,5}=2.9 \mathrm{~Hz}, \mathrm{H}-4$ ), 2.81 (dd, $1 \mathrm{H}$, $J_{5,6}=5.4 \mathrm{~Hz}, \mathrm{H}-6$ ), 2.59 (dd, $1 \mathrm{H}, J_{6,6^{\prime}}=14.4 \mathrm{~Hz}, \mathrm{H}-6^{\prime}$ ), 1.50 (s, $3 \mathrm{H}, \mathrm{CMe}$ ).

The mixture of above cyclohexanones $(19 \alpha$ and $19 \beta)$ $(0.77 \mathrm{~g}, 1.7 \mathrm{mmol})$ was treated with acetic anhydride $(3.0$ $\left.\mathrm{cm}^{3}\right)$ in pyridine $\left(20 \mathrm{~cm}^{3}\right)$ at r.t. for $12 \mathrm{~h}$ to give the corresponding enone derivative 20 in $94 \%$ yield $(0.68 \mathrm{~g})$, which was purified on a column of silica gel (hexane/ethyl acetate $=8 / 1$ ). 20: $\mathrm{Mp} 60.5{ }^{\circ} \mathrm{C}$ (recrystallized from ether-hexane); $[\alpha]_{\mathrm{D}}^{25}+85^{\circ}\left(\right.$ c $\left.0.95, \mathrm{CHCl}_{3}\right)$; IR $1689 \mathrm{~cm}^{-1}$ $(\mathrm{C}=\mathrm{O}$ and $\mathrm{C}=\mathrm{C}) ;{ }^{1} \mathrm{HNMR}$ (FX-200) $\delta=7.52-7.14(\mathrm{~m}$, $5 \mathrm{H} \times 3, \mathrm{Ph} \times 3$ ), 6.83 (ddd, $1 \mathrm{H}, J_{4,5}=2.2 \mathrm{~Hz}, J_{5,6}=10.3 \mathrm{~Hz}, \mathrm{H}-$ 5), 6.03 (ddd, $\left.1 \mathrm{H}, J_{4,6}=2.2 \mathrm{~Hz}, \mathrm{H}-6\right), 4.90-4.60(\mathrm{~m}, 2 \mathrm{H} \times 3$, $\left.\mathrm{PhCH}_{2}-\times 3\right), 4.31$ (dd, $\left.1 \mathrm{H}, \mathrm{H}-4\right), 4.20\left(\mathrm{~d}, 1 \mathrm{H}, J_{3,4}=8.1 \mathrm{~Hz}\right.$ $\mathrm{H}-3), 1.47$ (s, 3H, C-Me). Found: C, 77.96; H, 6.98\%. Calcd for $\mathrm{C}_{28} \mathrm{H}_{28} \mathrm{O}_{4}$ : C, $78.48 ; \mathrm{H}, 6.59 \%$.

1D-(1,3/2,4)-2,3,4-Tri- $O$-benzyl-2- $C$-methyl-5-cyclohexene-1,2,3,4-tetrol (21). The selective reduction of carbonyl group of 20 (1.0 g, $2.3 \mathrm{mmol})$ with cerium(III) chloride $\left(\mathrm{CeCl}_{3} \cdot 7 \mathrm{H}_{2} \mathrm{O}, 1.3 \mathrm{~g}, 3.5 \mathrm{mmol}, 1.5\right.$ equiv) and sodium tetrahydroborate $(0.16 \mathrm{~g}, 4.2 \mathrm{mmol}, 1.8$ equiv) in a mixed solvent (dichloromethane/ethanol $=1 / 2,21 \mathrm{~cm}^{3}$ ) was carried out at $-78{ }^{\circ} \mathrm{C}$ under argon in a similar manner as described regarding the reaction of $\mathbf{9}$, to give the corresponding cyclohexene derivative 21, which was purified on a column of silica gel (hexane/ethyl acetate $=3 / 1)$ in $91 \%$ yield $(0.91$ g). 21: $\mathrm{Mp} 74-75^{\circ} \mathrm{C} ;[\alpha]_{\mathrm{D}}^{22}+69^{\circ}\left(c, 1.0, \mathrm{CHCl}_{3}\right)$; IR $3448(\mathrm{O}-\mathrm{H})$ and $1605 \mathrm{~cm}^{-1}(\mathrm{C}=\mathrm{C}) ;{ }^{1} \mathrm{H}$ NMR $(\mathrm{FX}-200) \delta=$ $7.30-7.25(\mathrm{~m}, 5 \mathrm{H} \times 3, \mathrm{Ph} \times 3), 5.79\left(\mathrm{ddd}, 1 \mathrm{H}, J_{6,5}=10.3 \mathrm{~Hz}\right.$, $J_{6,1}=J_{6,4}=2.4 \mathrm{~Hz}, \mathrm{H}-6$ ), 5.70 (ddd, $1 \mathrm{H}, J_{5,1}=J_{5,4}=2.2 \mathrm{~Hz}$, $\mathrm{H}-5), 4.94\left(\mathrm{ABq}, 2 \mathrm{H}, J_{\mathrm{A}, \mathrm{B}}=11.2 \mathrm{~Hz}, \mathrm{PhCH}_{2}-\right), 4.81(\mathrm{ABq}$, $\left.2 \mathrm{H}, J_{\mathrm{A}, \mathrm{B}}=11.7 \mathrm{~Hz}, \mathrm{PhCH}_{2}-\right), 4.67\left(\mathrm{~s}, 2 \mathrm{H}, \mathrm{PhCH}_{2}-\right), 4.46$ (m, 1H, H-1), $4.15\left(\mathrm{~m}, 1 \mathrm{H}, J_{4,3}=7.3 \mathrm{~Hz}, \mathrm{H}-4\right), 3.84(\mathrm{~d}, 1 \mathrm{H}$, H-3), 1.34 (s, 3H, C-Me). Found: C, 78.39; H, 7.20\%. Calcd for $\mathrm{C}_{28} \mathrm{H}_{30} \mathrm{O}_{4}$ : C, $78.11 ; \mathrm{H}, 7.02 \%$.

Acetyl Derivative of 21: 1D- $(1,3 / 2,4)-1$-O-Acetyl2,3,4-tri- $O$-benzyl-2- $C$-methyl-5-cyclohexene-1,2,3,4-tetrol was prepared in order to confirm the structure of $\mathbf{2 1}$ in the usual acetylation by the use of acetic anhydride and pyridine. ${ }^{1} \mathrm{H}$ NMR $(\mathrm{FX}-200) \delta=7.36-7.25(\mathrm{~m}, 5 \mathrm{H} \times 3, \mathrm{Ph} \times 3)$, 5.82 (ddd, $\left.1 \mathrm{H}, J_{1,6}=2.4 \mathrm{~Hz}, \mathrm{H}-6\right), 5.73\left(\mathrm{~d}, 1 \mathrm{H}, J_{4,6}=2.0 \mathrm{~Hz}\right.$, $\mathrm{H}-1$ ), 5.52 (ddd, $1 \mathrm{H}, J_{5,1}=2.2 \mathrm{~Hz}, J_{5,6}=10.3 \mathrm{~Hz}, \mathrm{H}-5$ ), $4.93-$ $4.80\left(\mathrm{ABq}, 2 \mathrm{H}, \mathrm{PhCH}_{2}-\right), 4.68\left(\mathrm{~s}, 2 \mathrm{H}, \mathrm{PhCH}_{2}-\right), 4.68-4.54$ ( $\mathrm{ABq}, 2 \mathrm{H}, \mathrm{PhCH}_{2}-$ ), 4.12 (ddd, $1 \mathrm{H}, J_{4,5}=2.2 \mathrm{~Hz}, \mathrm{H}-4$ ) , 3.88 (d, $1 \mathrm{H}, J_{3,4}=7.8 \mathrm{~Hz}, \mathrm{H}-3$ ), 2.08 (s, 3H, OAc), 1.38 (s, 3H, $\mathrm{C}-\mathrm{Me})$.

1D-(1,3/2,4)-1,2,3,4-Tetra- $O$-benzyl-2- $C$-methyl-5cyclohexene-1,2,3,4-tetrol (22). A mixture of 21 (0.47 $\mathrm{g}, 1.1 \mathrm{mmol})$, sodium hydride [0.14 $\mathrm{g}$ ( $55 \%$ in oil), $3.3 \mathrm{mmol}$, 3.0 equiv], and benzyl bromide $(0.58 \mathrm{~g}, 3.4 \mathrm{mmol}, 3.1$ equiv) was treated in a similar manner as described regarding the benzylation of 7 to give the corresponding tetra- $O$-benzylated derivative $\mathbf{2 2}$ in quantitative yield, which was purified on a column of silica gel (hexane/ethyl acetate $=6 / 1$ ). 22: Syrup; $[\alpha]_{\mathrm{D}}^{25}+100^{\circ}\left(c 0.65, \mathrm{CHCl}_{3}\right) ;{ }^{1} \mathrm{H}$ NMR $(\mathrm{FX}-200) \delta=$ 7.35-7.22 (m, $5 \mathrm{H} \times 4, \mathrm{Ph} \times 4), 5.71(\mathrm{~m}, 2 \mathrm{H}, \mathrm{H}-5$ and $\mathrm{H}-6)$, $4.88-4.63\left(\mathrm{~m}, 2 \mathrm{H} \times 4, \mathrm{PhCH}_{2}-\times 4\right), 4.25\left(\mathrm{~d}, 1 \mathrm{H}, J_{1,6}=2.4\right.$ $\mathrm{Hz}, \mathrm{H}-1), 4.10$ (ddd, $\left.1 \mathrm{H}, J_{4,5}=2.4 \mathrm{~Hz}, \mathrm{H}-4\right), 3.78$ (d, $1 \mathrm{H}$, $\left.J_{3,4}=8.1 \mathrm{~Hz}, \mathrm{H}-3\right), 1.44(\mathrm{~s}, 3 \mathrm{H}, \mathrm{C}-\mathrm{Me})$. Found: C, 80:65; H, 6.94\%. Calcd for $\mathrm{C}_{35} \mathrm{H}_{36} \mathrm{O}_{4}: \mathrm{C}, 80.74 ; \mathrm{H}, 6.97 \%$.

1D-3,4,5,6-Tetra- $O$-benzyl-4- $C$-methyl-1,2,3,5/4,6cyclohexanehexol $(23)$ and $1 \mathrm{D}-3,4,5,6$-Tetra- $O$-benzyl-5- $C$-methyl-1,2,3,5/4,6-cyclohexanehexol (24). To a mixture of $22(0.33 \mathrm{~g}, 0.63 \mathrm{mmol})$ and 4-methylmorpholine $N$-oxide $(0.12 \mathrm{~g}, 0.96 \mathrm{mmol}, 1.5$ equiv $)$ in a mixed solvent (2-methyl-2-propanol/water $=4 / 1,15 \mathrm{~cm}^{3}$ ), osmium tetraoxide ( $0.1 \mathrm{~mol} \mathrm{dm}^{-3}$ 2-methyl-2-propanol solution, 3.0 $\mathrm{cm}^{3}$ ) was added and kept for $24 \mathrm{~h}$ under ultrasound until the disappearance of $\mathbf{2 2}$; sodium hydrogensulfite $(2.0 \mathrm{~g})$ was then added and stirred for $30 \mathrm{~min}$. The reaction mixture was filtered, poured into water, and extracted with chloroform. The organic layer was washed with water, dried over anhydrous magnesium sulfate, and evaporated to give a mixture of the corresponding cis-diol derivatives 23 and $\mathbf{2 4}$ in a ratio of $3: 5$. They were purified on a column of silica gel (hexane/ethyl acetate $=3 / 1$ ), but could not be separated. The total yield of $\mathbf{2 3}$ and $\mathbf{2 4}$ was $0.30 \mathrm{~g}(84 \%)$. The structures of 23 and 24 were confirmed by acetylation as follows. The mixture of $\mathbf{2 3}$ and $\mathbf{2 4}$ was acetylated in the usual manner to give the corresponding acetyl derivatives in quantitative yield, which also could not be separated.

Acetyl Derivative of 23: ${ }^{1} \mathrm{H}$ NMR (FX-200) $\delta=$ $7.35-7.26(\mathrm{~m}, 5 \mathrm{H} \times 4, \mathrm{Ph} \times 4), 5.75\left(\mathrm{dd}, 1 \mathrm{H}, J_{2,1}=3.4 \mathrm{~Hz}\right.$, $\mathrm{H}-2), 5.02-4.39\left(\mathrm{~m}, 1 \mathrm{H}\right.$ and $\left.2 \mathrm{H} \times 4, \mathrm{H}-1, \mathrm{PhCH}_{2}-\times 4\right), 3.83$ (dd, $\left.1 \mathrm{H}, J_{6,1}=10.3 \mathrm{~Hz}, J_{6,5}=10.0 \mathrm{~Hz}, \mathrm{H}-6\right), 3.71(\mathrm{~d}, 1 \mathrm{H}$, $J_{3,2}=3.2 \mathrm{~Hz}, \mathrm{H}-3$ ), 3.62 (d, 1H, H-5), 2.11 and 1.96 (each s, $3 \mathrm{H} \times 2, \mathrm{OAc} \times 2)$.

Acetyl Derivative of 24: ${ }^{1}$ HNMR (FX-200) $\delta=$ $7.35-7.26(\mathrm{~m}, 5 \mathrm{H} \times 4, \mathrm{Ph} \times 4), 5.72\left(\mathrm{dd}, 1 \mathrm{H}, J_{2,1}=J_{2,3}=\right.$ $2.9 \mathrm{~Hz}, \mathrm{H}-2), 5.02-4.39(\mathrm{~m}, 1 \mathrm{H}$ and $2 \mathrm{H} \times 4, \mathrm{H}-1$ and $\left.\mathrm{PhCH}_{2}-\times 4\right), 3.97$ (d, $\left.1 \mathrm{H}, J_{6,1}=10.7 \mathrm{~Hz}, \mathrm{H}-6\right), 3.94$ (d, $1 \mathrm{H}$, $J_{4,3}=10.0 \mathrm{~Hz}, \mathrm{H}-4$ ), 3.57 (dd, $1 \mathrm{H}, \mathrm{H}-3$ ), 2.17 and 1.94 (each $\mathrm{s}, 3 \mathrm{H} \times 2, \mathrm{OAc} \times 2), 1.61(\mathrm{~s}, 3 \mathrm{H}, \mathrm{C}-\mathrm{Me})$.

1D-1,3,4,5,6-Penta- $O$-benzyl-4- $C$-methyl-1,2,3,5/4, 6-cyclohexanehexol (25) and 1D-1,3,4,5,6-Penta$O$-benzyl-5- $C$-methyl-1,2,3,5/4,6-cyclohexanehexol (26). A mixture of compounds (23 and 24) $(0.27 \mathrm{~g}$, $0.49 \mathrm{mmol})$ and dibutyltin oxide $(0.18 \mathrm{~g}, 0.74 \mathrm{mmol}, 1.5$ equiv) in toluene $\left(9.0 \mathrm{~cm}^{3}\right)$ was refluxed for $2 \mathrm{~h}$; a solution of benzyl bromide $(0.86 \mathrm{~g}, 5.0 \mathrm{mmol})$ in $\operatorname{DMF}\left(4.0 \mathrm{~cm}^{3}\right)$ 
was then added dropwise and stirred at $80^{\circ} \mathrm{C}$ for $3 \mathrm{~h}$ until the disappearance of the starting materials. To the reaction mixture, sodium methoxide and potassium fluoride solution were added consecutively. The reaction mixture was stirred for $30 \mathrm{~min}$., then filtered, poured into water, extracted with ethyl acetate, dried over anhydrous magnesium sulfate, and evaporated to give $\mathbf{2 5}$ and $\mathbf{2 6}$, which were purified on a column of silica gel (hexane/ethyl acetate=9/1), in $92 \%(0.11$ g) and $74 \%(0.15 \mathrm{~g})$ yields, respectively.

25: Syrup; IR $3480 \mathrm{~cm}^{-1}(\mathrm{OH}) ;[\alpha]_{\mathrm{D}}^{25}-1.3^{\circ}$ (c 1.1, $\mathrm{CHCl}_{3}$ ). Found: C, $78.58 ; \mathrm{H}, 6.86 \%$. Calcd for $\mathrm{C}_{42} \mathrm{H}_{44} \mathrm{O}_{6}$ : C, $78.23 ; \mathrm{H}, 6.88 \%$.

Acetyl Derivative of 25: ${ }^{1} \mathrm{H}$ NMR (FX-200) $\delta=$ $7.46-7.24(\mathrm{~m}, 5 \mathrm{H} \times 5, \mathrm{Ph} \times 5), 5.41\left(\mathrm{dd}, 1 \mathrm{H}, J_{2,3}=3.2 \mathrm{~Hz}, \mathrm{H}-\right.$ 2), $4.94-4.89\left(\mathrm{ABq}, 2 \mathrm{H}, \mathrm{PhCH}_{2}-\right), 4.81-4.72(\mathrm{~m}, 2 \mathrm{H} \times 4$, $\left.\mathrm{PhCH}_{2}-\times 4\right), 3.84$ (dd, $\left.1 \mathrm{H}, J_{6,5}=10.3 \mathrm{~Hz}, \mathrm{H}-6\right), 3.56(\mathrm{~d}, 1 \mathrm{H}$, $\mathrm{H}-3$ ), 3.50 (d, 1H, H-5), 3.46 (dd, $1 \mathrm{H}, J_{1,2}=7.4 \mathrm{~Hz}, \mathrm{H}-1$ ), 2.14 (s, 3H, OAc), 1.60 (s, 3H, C-Me).

26 (meso): $\mathrm{Mp} 114-115^{\circ} \mathrm{C}$ (recrystallized from ethanol-hexane); IR $3478 \mathrm{~cm}^{-1}(\mathrm{O}-\mathrm{H}) ;{ }^{1} \mathrm{H}$ NMR (FX-200) $\delta=7.52-7.21(\mathrm{~m}, 5 \mathrm{H} \times 5, \mathrm{Ph} \times 5), 4.86(\mathrm{ABq} \times 2,2 \mathrm{H} \times 2$, $\left.\mathrm{PhCH}_{2}-\times 2\right), 4.76\left(\mathrm{~s}, 2 \mathrm{H}, \mathrm{PhCH}_{2}-\right), 4.70(\mathrm{~s} \times 2,2 \mathrm{H} \times 2$, $\left.\mathrm{PhCH}_{2}-\times 2\right), 4.23\left(\mathrm{dd}, 1 \mathrm{H}, J_{2,3}=J_{2,1}=2.9 \mathrm{~Hz}, \mathrm{H}-2\right), 4.09$ (d, $2 \mathrm{H}, J_{4,3}=J_{6,1}=9.8 \mathrm{~Hz}, \mathrm{H}-4$ and $\left.\mathrm{H}-6\right), 3.39$ (dd, $2 \mathrm{H}, \mathrm{H}-3$ and $\mathrm{H}-1), 2.58$ (bs, $1 \mathrm{H}, \mathrm{OH}), 1.41(\mathrm{~s}, 3 \mathrm{H}, \mathrm{C}-\mathrm{Me})$. Found: $\mathrm{C}$, $78.58 ; \mathrm{H}, 6.86 \%$. Calcd for $\mathrm{C}_{42} \mathrm{H}_{44} \mathrm{O}_{6}: \mathrm{C}, 78.23 ; \mathrm{H}, 6.88 \%$.

Acetyl Derivative of 26: ${ }^{1} \mathrm{H}$ NMR $(\mathrm{FX}-200) \delta=$ $7.32-7.19(\mathrm{~m}, 5 \mathrm{H} \times 5, \mathrm{Ph} \times 5), 5.87\left(\mathrm{dd}, 1 \mathrm{H}, J_{2,3}=10.3 \mathrm{~Hz}\right.$, $\mathrm{H}-2), 4.96-4.90\left(\mathrm{ABq}, 2 \mathrm{H}, \mathrm{PhCH}_{2}-\right), 4.79-4.74(\mathrm{~m}, 2 \mathrm{H} \times 4$, $\left.\mathrm{PhCH}_{2}-\times 4\right), 3.94\left(\mathrm{~d}, 2 \mathrm{H}, J_{4,3}=J_{6,1}=10.3 \mathrm{~Hz}, \mathrm{H}-4\right.$ and $\mathrm{H}-$ 6), $3.46\left(\mathrm{dd}, 2 \mathrm{H}, J_{1,2}=3.2 \mathrm{~Hz}, \mathrm{H}-1\right.$ and $\left.\mathrm{H}-3\right), 2.19$ (s, $3 \mathrm{H}$, $\mathrm{OAc}), 1.44$ (s, 3H, C-Me).

Peracetyl Derivative of (-)-Laminitol (3) (1DHexa- $O$-acetyl-4- $C$-methyl-1,2,3,5/4,6-cyclohexanehexol). Compound 25 was hydrogenolyzed in the presence of $10 \% \mathrm{Pd} / \mathrm{C}$ to give the corresponding laminitol $\mathbf{3}$ in quantitative yield. Then, $\mathbf{3}$ was acetylated with acetic anhydride and catalytic amount of 4-dimethylaminopyridine in pyridine at $40^{\circ} \mathrm{C}$ for $12 \mathrm{~h}$ to give the corresponding peracetylated derivative in quantitative yield, the physical data of which were identical with that reported. ${ }^{18)}$

Peracetyl Derivative of (-)-Laminitol 3: Mp 151$152^{\circ} \mathrm{C}$; IR $1758 \mathrm{~cm}^{-1}(\mathrm{C}=\mathrm{O}) ;[\alpha]_{\mathrm{D}}^{25}-18^{\circ}$ (c $\left.0.37, \mathrm{CHCl}_{3}\right)$; $\left[\right.$ lit $\left.{ }^{18)} \mathrm{Mp} 151-152{ }^{\circ} \mathrm{C} ;[\alpha]_{\mathrm{D}}-19^{\circ}\left(c 2.0, \mathrm{CHCl}_{3}\right)\right] ;{ }^{1} \mathrm{H}$ NMR $(\mathrm{FX}-200) \delta=6.37\left(\mathrm{~d}, 1 \mathrm{H}, J_{3,2}=3.9 \mathrm{~Hz}, \mathrm{H}-3\right), 6.19(\mathrm{~d}, 1 \mathrm{H}$, $\left.J_{5,6}=10.0 \mathrm{~Hz}, \mathrm{H}-5\right), 5.69$ (dd, $\left.1 \mathrm{H}, J_{2,1}=3.4 \mathrm{~Hz}, \mathrm{H}-2\right), 5.51$ (dd, $1 \mathrm{H}, J_{6,1}=10.5 \mathrm{~Hz}, \mathrm{H}-6$ ) $, 2.25,2.16,2.15,2.11,2.07$, and 2.00 (each s, $3 \mathrm{H} \times 6, \mathrm{OAc} \times 6), 1.63$ (s, $3 \mathrm{H}, \mathrm{C}-\mathrm{Me})$. Found: C, $51.29 ; \mathrm{H}, 5.66 \%$. Calcd for $\mathrm{C}_{19} \mathrm{H}_{26} \mathrm{O}_{12}$ : C, $51.12 ; \mathrm{H}, 5.87 \%$.

1D-1,3,4,5,6-Penta- $O$-benzyl-2- $O$-methylsulfonyl-5$C$-methyl-1,2,3,5/4,6-cyclohexanehexol (27). Methanesulfonylation of $26(0.23 \mathrm{~g}, 0.36 \mathrm{mmol})$ with methanesulfonyl chloride $(0.76 \mathrm{~g}, 6.6 \mathrm{mmol}, 18$ equiv) in pyridine (5.0 $\mathrm{cm}^{3}$ ) at $0{ }^{\circ} \mathrm{C}$ for $24 \mathrm{~h}$ under ultrasound gave 27 in quantitative yield on TLC. The reaction mixture was treated with methanol, evaporated, and purified on a column of silica gel (hexane/ethyl acetate $=1 / 1$ ) to give $0.25 \mathrm{~g}(97 \%)$ of $\mathbf{2 7}$.

27 (meso): Syrup; IR: 1455 and $1179 \mathrm{~cm}^{-1}(\mathrm{~S}=\mathrm{O})$; ${ }^{1} \mathrm{HNMR}(\mathrm{FX}-200) \delta=7.60-7.25(\mathrm{~m}, 5 \mathrm{H} \times 5, \mathrm{Ph} \times 5), 5.36$ (dd, $\left.1 \mathrm{H}, J_{2,1}=J_{2,3}=2.7 \mathrm{~Hz}, \mathrm{H}-2\right), 4.95-4.60(\mathrm{~m}, 2 \mathrm{H} \times 5$, $\mathrm{PhCH}_{2}-\times 5$ ), 3.94 (d, 2H, $J_{4,3}=J_{6,1}=10.0 \mathrm{~Hz}, \mathrm{H}-4$ and $\mathrm{H}-6$ ), 3.44 (dd, 2H, H-1 and $\mathrm{H}-3), 3.03$ (s, 3H, OMs), 1.43 (s, 3H,
C-Me). Found: C, $71.61 ; \mathrm{H}, 6.36 \%$. Calcd for $\mathrm{C}_{43} \mathrm{H}_{46} \mathrm{O}_{8} \mathrm{~S}$ : $\mathrm{C}, 71.45 ; \mathrm{H}, 6.42 \%$.

1D-1- $O$-Acetyl-2,3,4,5,6-penta- $O$-benzyl-4- $C$-methyl-1,3,5/2,4,6-cyclohexanehexol (28). A mixture of $27(68 \mathrm{mg}, 0.094 \mathrm{mmol})$ and cesium acetate $(0.18 \mathrm{~g}, 0.94$ mmol, 10 equiv) in DMF $\left(1.5 \mathrm{~cm}^{3}\right)$ was stirred at $80^{\circ} \mathrm{C}$ until the disappearance of $\mathbf{2 7}$ (for $12 \mathrm{~h}$ ). The reaction mixture was poured into water, extracted with ethyl acetate, dried over anhydrous magnesium sulfate, and evaporated to give $60 \mathrm{mg}(93 \%)$ of the corresponding acetate 28, which was recrystallized from ethanol-hexane.

28 (meso): $\quad \mathrm{Mp} 146-148{ }^{\circ} \mathrm{C}$; IR $1746 \mathrm{~cm}^{-1}(\mathrm{C}=\mathrm{O})$; ${ }^{1} \mathrm{HNMR}(\mathrm{FX}-200) \delta=7.36-7.21(\mathrm{~m}, 5 \mathrm{H} \times 5, \mathrm{Ph} \times 5), 5.20$ (dd, $\left.1 \mathrm{H}, J_{1,2}=J_{1,6}=9.8 \mathrm{~Hz}, \mathrm{H}-1\right), 3.69\left(\mathrm{~d}, 2 \mathrm{H}, J_{3,2}=J_{5,6}=\right.$ $10.0 \mathrm{~Hz}, \mathrm{H}-3$ and $\mathrm{H}-5$ ), 3.50 (dd, $2 \mathrm{H}, \mathrm{H}-2$ and $\mathrm{H}-6$ ), 1.83 (s, $3 \mathrm{H}, \mathrm{OAc}), 1.52(\mathrm{~s}, 3 \mathrm{H}, \mathrm{C}-\mathrm{Me}$ ). Found: $\mathrm{C}, 77.15 ; \mathrm{H}, 6.80 \%$. Calcd for $\mathrm{C}_{44} \mathrm{H}_{46} \mathrm{O}_{7}: \mathrm{C}, 76.94 ; \mathrm{H}, 6.75 \%$.

Peracetyl Derivative of Mytilitol (4) (1D-Hexa$O$-acetyl-4- $C$-methyl-1,3,5/2,4,6-cyclohexanehexol). To the above product $28(60 \mathrm{mg}, 0.087 \mathrm{mmol})$ in methanol $\left(1.0 \mathrm{~cm}^{3}\right)$, sodium methoxide was added dropwise at r.t. until the $\mathrm{pH}$ value 10 and stirred until the disappearance of 28 . The reaction mixture was then neutralized with ion-exchange resin (Dowex 50W-X8) and evaporated to give the deacetylated derivative in quantitative yield. Hydrogenolysis of the above product in the presence of $10 \% \mathrm{Pd} / \mathrm{C}$ in methanol $\left(3.0 \mathrm{~cm}^{3}\right)$ and the usual work up of the reaction mixture gave the corresponding mytilitol $\mathbf{4}$ in quantitative yield. The structure of $\mathbf{4}$ was confirmed by peracetylation [acetic anhydride $\left(0.5 \mathrm{~cm}^{3}\right)$ and catalytic amount of 4-dimethylaminopyridine in pyridine $\left(2.0 \mathrm{~cm}^{3}\right)$ at $40{ }^{\circ} \mathrm{C}, 12 \mathrm{~h}$ ]. The product was recrystallized from ethanol-hexane and the yield of hexa- $O$-acetylated derivative of $\mathbf{4}$ from $\mathbf{2 8}$ was 36 $\mathrm{mg}(92 \%)$.

Peracetyl Derivative of Mytilitol 4 (meso): Mp 181 ${ }^{\circ} \mathrm{C}$; [lit $\left.{ }^{19)} \mathrm{mp} 181{ }^{\circ} \mathrm{C}\right] ;{ }^{1} \mathrm{H}$ NMR (FX-200) $\delta=6.17$ (d, 2H, $J_{3,2}=J_{5,6}=9.3 \mathrm{~Hz}, \mathrm{H}-3$ and H-5), 5.26 (dd, $2 \mathrm{H}, J_{2,1}=J_{6,1}=9.9$ $\mathrm{Hz}, \mathrm{H}-2$ and $\mathrm{H}-6$ ), 5.24 (dd, $1 \mathrm{H}, \mathrm{H}-1$ ), 2.06 (each s, $3 \mathrm{H} \times 2$, $\mathrm{OAc} \times 2), 2.01$ (each s, $3 \mathrm{H} \times 3, \mathrm{OAc} \times 3), 1.90(\mathrm{~s}, 3 \mathrm{H}, \mathrm{OAc})$, $1.49(\mathrm{~s}, 3 \mathrm{H}, \mathrm{C}-\mathrm{Me})$. Found: $\mathrm{C}, 51.52 ; \mathrm{H}, 5.88 \%$. Calcd for $\mathrm{C}_{19} \mathrm{H}_{26} \mathrm{O}_{12}: \mathrm{C}, 51.12 ; \mathrm{H}, 5.87 \%$.

\section{References}

1) A. A. Abldel-Latif, Pharmacol. Rev., 38, 227 (1986); R. H. Michell, Nature, 324, 613 (1986).

2) J. R. Falck, A. Abdali, and S. J. Wittenberger, J. Chem. Soc., Chem. Commun., 1990, 953; D. Lampe and B. V. L. Potter, J. Chem. Soc., Chem. Commun., 1990, 1500.

3) L. Anderson, "The Carbohydrates. Vol. 1A: Chemistry and Biochemistry," ed by W. Pigman and D. Horton, Academic Press (1972) Chap. 15, p. 519.

4) W. H. Schopfer and T. Posternak, Helv. Chim. Acta, 43, 2147 (1960).

5) S. J. Angyal, J. E. Klavins, and J. A. Mills, Aust. J. Chem., 27, 1075 (1974).

6) H. A. J. Carless and O. Z. Oak, Tetrahedron Lett., 32, 1671 (1991).

7) D. C. Billington, Chem. Soc. Rev., 18, 83 (1989).

8) W. Tegge and C. E. Ballou, Proc. Natl. Acad. Sci. U. S. A., 86, 94 (1989); T. Akiyama, N. Takechi, and S. Ozaki, Tetrahedron Lett., 31, 1433 (1990); A. P. Kozikowski, A. H. 
Fauq, I. A. Aksoy, M. J. Seewald, and G. Powis, J. Am Chem. Soc., 112, 7403 (1990); M. J. Seewald, I. A. Aksoy, G. Powis, A. H. Fauq, and A. P. Kozikowski, J. Chem. Soc., Chem. Commun., 1990, 1638.

9) J. R. Falck and P. Yadagiri, J. Org. Chem., 54, 5851 (1989); K. K. Reddy, J. R. Falck, and J. Capdevila, Tetrahedron Lett., 34, 7869 (1993).

10) K. Sato, S. Sakuma, S. Muramatsu, and M. Bokura, Chem. Lett., 1991, 1473.

11) Y. Watanabe, M. Mitani, and S. Ozaki, Chem. Lett., $1987,123$.

12) K. Sato, S. Sakuma, Y. Nakamura, J. Yoshimura, and H. Hashimoto, Chem. Lett., $1991,17$.

13) A. J. Mancuso, S. L. Huang, and D. Swern, J. Org. Chem., 43, 2480 (1978).
14) K. Sato, N. Kubo, R. Takada, and S. Sakuma, Bull. Chem. Soc. Jpn., 66, 1156 (1993).

15) R. J. Ferrier, J. Chem. Soc., Perkin Trans. 1, 1979, 1455; H. B. Mereyala and S. Guntha, J. Chem. Soc., Perkin Trans. 1, 1993, 841; C. Jaramillo and M. M. -Lomas, Tetrahedron Lett., 32, 2501 (1991); S. L. Bender and R. J. Budhu, J. Am. Chem. Soc., 113, 9883 (1991); V. A. Estevez and G. D. Prestwich, J. Am. Chem. Soc., 113, 9885 (1991).

16) S. Ozaki, Y. Watanabe, T. Ogasawara, Y. Kondo, N. Shiotani, H. Nishii, and T. Matsuki, Tetrahedron Lett., 27, 3157 (1986).

17) K. Sato, K. Kubo, N. Hong, H. Kodama, and J. Yoshimura, Bull. Chem. Soc. Jpn., 55, 938 (1982).

18) T. Posternak, Helv. Chim. Acta., 44, 2080 (1962).

19) T. Posternak, Helv. Chim. Acta., 27, 457 (1944). 